\title{
Automatic quantity processing in 5-year olds and adults
}

\author{
Titia Gebuis · Roi Cohen Kadosh · Edward de Haan · \\ Avishai Henik
}

Received: 7 February 2008/Revised: 26 May 2008/Accepted: 27 May 2008/Published online: 8 July 2008

(C) The Author(s) 2008

\begin{abstract}
In this study adults performed numerical and physical size judgments on a symbolic (Arabic numerals) and non-symbolic (groups of dots) size congruity task. The outcomes would reveal whether a size congruity effect (SCE) can be obtained irrespective of notation. Subsequently, 5-year-old children performed a physical size judgment on both tasks. The outcomes will give a better insight in the ability of 5-year-olds to automatically process symbolic and non-symbolic numerosities. Adult performance on the symbolic and non-symbolic size congruity tasks revealed a SCE for numerical and physical size judgments, indicating that the non-symbolic size congruity task is a valid indicator for automatic processing of nonsymbolic numerosities. Physical size judgments on both tasks by children revealed a SCE only for non-symbolic notation, indicating that the lack of a symbolic SCE is not related to the mathematical or cognitive abilities required for the task but instead to an immature association between the number symbol and its meaning.
\end{abstract}

T. Gebuis $(\bowtie) \cdot$ E. de Haan

Department of Experimental Psychology, Helmholtz Institute, Utrecht University, Heidelberglaan 2, 3584 CS Utrecht,

The Netherlands

e-mail: T.Gebuis@uu.nl

R. Cohen Kadosh

Institute of Cognitive Neuroscience, University College London, London, UK

\section{A. Henik}

Department of Behavioral Sciences and Zlotowski Center for Neuroscience, Ben-Gurion University of the Negev, Beersheba, Negev, Israel
Keywords Numerical cognition - Numerical Stroop task · Children · Automatic

\section{Introduction}

In the last decades, evidence has accumulated that numbers may be presented in the mind as an "inner number line" (e.g., logarithmic curve, Dehaene 2003, or linear curve with a scalar variability, Gallistel and Gelman 1992; Zorzi and Butterworth 1999, but see Verguts et al. 2005, for a different opinion). On this mental number line, small numbers are represented on the left while large numbers are represented on the right. The effect supporting this theory is the Spatial-Numerical Association of Response Codes (SNARC) effect, which relates to the phenomenon that people are faster to respond to numerically small numbers with the left hand and to numerically large numbers with the right hand (Dehaene et al. 1993; see Fischer et al. 2004; Gevers, Lammertyn, Notebaert, Verguts, and Fias 2006, for recent reviews). It has been suggested that the mapping of Arabic numerals on the mental number line happens automatically (Dehaene 1992). Strong evidence for the automatic processing of numbers (e.g., the meaning of the number symbol is directly accessed although it is not part of a task requirement) has been derived from the size congruity task (Algom et al. 1996; Cohen Kadosh et al. 2007a, b; Henik and Tzelgov 1982; Kaufmann et al. 2006; Schwarz and Heinze 1998; Szucs and Soltesz 2007; Szucs et al. 2007; Tzelgov et al. 1992).

In the size congruity task participants perceive two stimuli that are varied in their numerical value and physical size throughout the trials. In the size comparison task, participants have to decide which number is physically 
larger while ignoring the numerical value. In the numerical comparison task they have to decide which number is numerically larger while ignoring the physical size. Due to the different sizes and numbers used, three conditions can be distinguished: (1) a congruent condition where the numerically larger number is also physically larger (e.g., 1-4), (2) an incongruent condition where the numerically larger number is physically smaller (e.g., 1-4) and (3) a neutral condition where, in the size comparison task, the same numbers are presented in distinct sizes (e.g., 4-4) or where, in the numerical comparison task, different numbers are presented in the same size (e.g., 1-4). Performance on the task is influenced by the ability to ignore the irrelevant dimension which is reflected in the reaction times. The reaction times in the congruent condition are shorter (facilitation effect), whereas the reaction times in the incongruent condition are longer (interference effect) when compared to the neutral condition. The difference between the incongruent and congruent condition is called the size congruity effect (SCE) and reflects the integration of both dimensions.

At which age can we process numerical magnitude automatically? Girelli et al. (2000) used the size congruity task to get more insight into the development of automatic number processing in children. When children have to judge physical size, a SCE emerged in third grade children (mean age 8.3 years). This finding is comparable with the results of Rubinsten et al. (2002) who found a SCE in first grade children (mean age 7.32 years). But a recent study of Zhou et al. (2007) revealed a SCE already in Chinese kindergartners (mean age 5.8 years) and suggested that this was related to cross-cultural differences. Importantly, the children of the youngest age groups in the studies of $\mathrm{Ru}-$ binsten et al. (2002) and Girelli et al. (2000) did not reveal automatic mapping of numbers, but could still perfectly decide which number was numerically larger (Girelli et al. 2000; Rubinsten et al. 2002), indicating that these children did have knowledge of the number symbols. Therefore, these studies nicely demonstrate the distinction between intentional processing of numbers and automatic access to them.

We expect that the inability of 5-year-old children to process numbers automatically is related to an immature link between the number symbol and its meaning and not to the underlying mathematical or cognitive abilities required for the task. Support for our view comes from studies that reveal that 5-year-old children already have an understanding of the number symbols (Lipton and Spelke 2005) that is comparable to that of adults (Huntley-Fenner 2001; Temple and Posner 1998) and they can, as well as infants can, perform basic mathematical procedures with nonsymbolic stimuli, e.g., arrays of dots (Barth et al. 2003, 2006, 2005; Lipton and Spelke 2005; Brannon 2002;
Brannon et al. 2004; Feigenson 2005; Jordan and Brannon 2006; Mix et al. 2002; Xu and Spelke 2000; Mix et al. 2002, 2005). Note however, that Rousselle et al. (2004) disentangled the effect of each continuous variable (e.g., density, area, contour length) on numerosity judgment performance in 3-year-old children and revealed that they performed at chance level in the condition that controlled for surface area.

In the current study we aimed to gain further insight in the automatic processing of number symbols in 5-year-old children. We used children of this age group because they have understanding of the number symbols (HuntleyFenner 2001; Lipton and Spelke 2005; Temple and Posner 1998) but do not have automatic access to those number symbols yet (Girelli et al. 2000; Rubinsten et al. 2002). First, we intended to investigate whether a non-symbolic Stroop task (see Fig. 1) leads to a size congruity effect just like the symbolic Stroop task. Secondly, we intended to investigate automatic processing of symbolic and nonsymbolic numerosities in 5-year-old children. To this extent we looked at the performance of adults when they have to make a numerical or a physical size judgment on a symbolic and a non-symbolic size congruity task. Comparing the response patterns of the symbolic and nonsymbolic tasks gave us insight in the effect of continuous variables in the non-symbolic task, which leads to a better interpretation of the performance of the children on this task. For the non-symbolic task, groups of dots with a large numerosity were used to avoid the problem of differences in individuals subitizing range (the range up to which one can directly estimate the correct number presented). When a SCE is obtained in the non-symbolic task, it can be concluded that the non-symbolic task measures automatic numerosity processing. Subsequently, we investigated the performance of the 5-year-old children on both the symbolic and non-symbolic tasks by judging only physical size. Based on previous reports we do not expect a SCE on the symbolic task. If a SCE is present on the non-symbolic task, it can be concluded that children have direct access to non-symbolic numerosities although it is not part of the
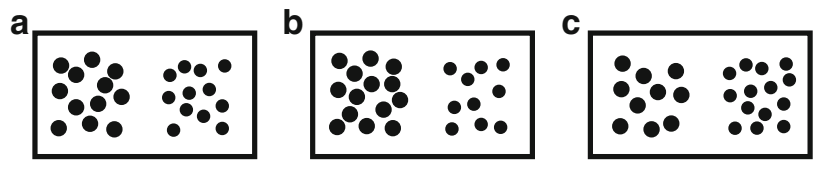

Fig. 1 Stimuli presented in the non-symbolic task. a Neutral condition of the physical size comparison task; the number of dots is the same but the physical size differs. b Congruent condition; more and physically larger dots have to be compared to less and physically smaller dots that together have a smaller total surface area (i.e., luminance) c Incongruent condition; more but physically smaller dots have to be compared to less but physically larger dots that together have a larger total surface area (i.e., luminance) 
task requirement. Moreover, the presence of a SCE on the non-symbolic and the absence of a SCE on the symbolic task indicate that 5-year-old children have an immature connection between the number symbol and its meaning and do not lack the mathematical abilities necessary to reveal a SCE on the task (i.e., knowledge about physical and numerical sizes, and the fact that they can be in conflict or agreement with each other).

\section{Methods}

In the adult experiment, the symbolic (Arabic numerals) and non-symbolic (groups of dots) tasks were performed by university students that had to make a numerical as well as a physical size comparison judgment. For the symbolic size congruity task participants were presented with two Arabic numerals that differed in their physical size and numerical value. In the physical size comparison task, subjects had to judge at which side the physically larger number was presented. In the numerical comparison task, subjects had to judge at which side the numerically larger number was presented. Similarly, in the non-symbolic size congruity tasks participants were presented arrays of dots and were instructed to judge which side contained physically larger dots (physical size comparison task) or which side contained more dots (numerical comparison task).

In the child study, the symbolic and non-symbolic size congruity tasks were performed by 5 -year-old children that only had to make a physical size judgment to investigate whether they can process symbolic and/or non-symbolic quantities automatically.

\section{Participants}

For the adults study, 16 students aged between 19 and 23 years $(M=20.7, \mathrm{SD}=1.4 ; 13$ female, 3 male $)$ from the University of Utrecht participated. All participants were native Dutch speakers and had normal or corrected-tonormal vision. The students were paid for their participation in this experiment.

For the child study, 16 children aged 5 years and 3 months to 5 years and 10 months $(M=5$ years and 7 months, 7 female, 9 male) from an elementary school in Utrecht participated. All participants were native Dutch speakers and had normal or corrected-to-normal vision. In order to keep the group as homogenous as possible, we only included children that had LVS scores (the school education system scores) of level 1 or 2 out of 5, meaning that their overall performance was average or well above average. It was tested in advance whether they had knowledge of the Arabic numerals 1-9 and their relations. The children had to judge which number was numerically larger for all the possible conditions that were used in the symbolic task. Only children that gave immediate responses (excluding children counting on the fingers or reciting the number line) and had $95 \%$ or more of the trials correct were included the in the study. Three children were excluded because they were unable to do so. The children afterward received a present for participation.

Stimuli and materials

\section{Symbolic comparison}

In each trial, two numbers were displayed simultaneously at $2.75^{\circ}$ at the right and left side from the center of the screen. The stimuli consisted of Arabic numbers ranging from 1 to 9 , which were presented in pairs with a small $(1-2,2-3,3-4,4-5,5-6,6-7,7-8,8-9)$ or large $(1-6,2-7$, $3-8,4-9)$ numerical distance in two different font sizes: $1.7^{\circ}$ (small) and $2.4^{\circ}$ (large).

\section{Non-symbolic comparison}

In each trial, groups of dots ranging from 11 to 20 were randomly distributed; this relatively large number of dots had been used to rule out possible subitizing effects. Again, two numerical distances were used, a small numerical distance of four $(11-15,12-16,13-17,14-18$, 15-19, 16-20) and a large numerical distance of seven $(11-18,12-19,13-20)$ and two dots sizes were used. Small dots had a diameter of $0.38^{\circ}$ and the large dots had a diameter of $0.53^{\circ}$. To exclude the possibility that the participant could derive the correct answer on the basis of visual sensory properties, we controlled for the area subtended by the group of dots presented in one array and the total surface area of the dots (i.e., luminance of the stimulus, similar to experiment 1 of the Hurewitz et al. (2006) study). The area subtended by the stimulus was the same in each condition (width and height of $3.05^{\circ}$ ). The dots were scattered randomly over the whole surface area and did not overlap. The total surface area of the dots was calculated by multiplying the surface area of one dot with the number of dots present in the stimulus. Thus, in the congruent condition an array of dots with more and physically larger dots (which together constitute a larger surface area), has to be compared with an array of dots with fewer and physically smaller dots (which together constitute a smaller surface area). In the incongruent condition an array with more but physically smaller dots (which together constitute a smaller surface area) is compared to an array with fewer but physically larger dots (which together constitute a larger surface area). In the neutral condition two arrays that contain the same number of dots are presented of which one array contains physically larger dots. 
Stimuli were presented on a 17 -inch computer screen. Stimuli were presented using the Presentation software (Neurobehavioral Systems).

\section{Procedure}

Two tasks were constructed in different blocks. In the symbolic experiment there were two different instruction conditions (physical size comparison and numerical comparison) and three congruency conditions (congruent, incongruent and neutral). Each congruency condition consisted of 32 trials (total of 192 trials per task). For the non-symbolic number comparison task it was not possible to create a neutral condition while taking into account the additional visual-sensory properties of the stimulus. When the number of dots varies while the physical size of the dots remains the same, it is inevitable that the side with the numerically more dots is darker and denser. These aspects of the stimulus serve as a marker for the side that contains more dots; the stimulus is not neutral. Therefore, the nonsymbolic number comparison task comprised two instruction conditions (physical size and numerical comparison) and only two congruency conditions (congruent and incongruent) while the non-symbolic size comparison task consisted of two instruction conditions (physical size and numerical comparison) and three congruency conditions (congruent, incongruent and neutral). The order of the tasks was counterbalanced between participants. Participants sat at a distance of approximately $57 \mathrm{~cm}$ and had to respond by pressing the button at the corresponding side of the target (the physically or numerically larger number). Half of the trials were presented with the correct answer on the left side and half of the trials with the correct answer on the right side. Before the experimental trials started, participants received instructions and performed ten practice trials. Between each task participants could take a break. Each trial began with a fixation point $(500 \mathrm{~ms})$, followed by the stimulus (until response) and an inter-trial interval (500 ms).

For the children only, the experimental tasks comprised ten cartoon pictures that were randomly presented throughout each experiment to keep them motivated. They were told that cartoon pictures would appear on the screen, the better and faster they performed the task.

\section{Analyses}

For each participant, median reaction times of the correct trials were calculated in each condition and used as a dependent variable in the four-way ANOVA with notation (symbolic and non-symbolic), order of task (physical size or numerical comparison first), task (physical or numerical comparison) and congruity (congruent and incongruent) as within participant factors. The median instead of the mean was used to deal with possible outliers. The neutral condition was not included in the overall repeated measures ANOVA, because this condition was not present in the non-symbolic numerical comparison task. In the case of interactions additional analyses were done. In this case, in order to examine whether the SCE was interference and/or facilitatory based, we compared the neutral condition to the congruent and the incongruent condition except for the non-symbolic numerical comparison task.

As mentioned in the methods section, the children only performed physical size judgments. Therefore a two-way ANOVA was conducted with notation (symbolic and nonsymbolic) and congruency (congruent, incongruent and neutral).

\section{Results of the adult study}

The four-way ANOVA revealed significant main effects for task $[F(1,14)=160.33, P<0.001]$ and congruency $[F(1,14)=124.37, P<0.001]$. The two-way interaction between task and congruency $[F(1,14)=68.60$, $P<0.001]$ and task and notation $[F(1,14)=20.29$, $P<0.005]$ and notation and congruency $[F(1,14)=8.51$, $P<0.03]$ were significant. No triple or four-way interactions were significant. To further our understanding regarding the source of the two-way interactions, we conducted simple effects analyses for numerical and size comparisons separately under notation (for the interaction between task and notation), and under congruency (for the interaction between task and congruency) (Keppel 1991).

\section{Numerical comparison}

The simple main effect for notation was significant for reaction time $[F(1,14)=4.61, P<0.05]$ but not for error rate $[F<1]$. This means that adults performed significantly slower but equally well in the non-symbolic (576 ms) compared to the symbolic numerical comparison task (492 ms). In addition, a simple main effect for congruency was present for both the reaction times $[F(1$, $14)=173.07, \quad P<0.001]$ and the error rate $[F(1$, $14)=21.544, P<0.001]$ (see Fig. 2). Adults were significantly slower and made more errors on the incongruent (579 $\mathrm{ms}, 9 \%)$ compared to the congruent trials $(488 \mathrm{~ms}$, $3 \%)$.

In order to examine whether the SCE was interference and/or a facilitatory based, we analyzed the congruency effect under symbolic notation for the symbolic numerical comparison task only because the non-symbolic condition did not consist of a neutral condition. A significant facilitation effect of $47 \mathrm{~ms}[F(1,14)=42.71, P<0.001]$ as 
Fig. 2 The performance of the adults on the symbolic and nonsymbolic size congruity tasks. a Mean reaction time on the symbolic and non-symbolic numerical comparison task, b mean reaction time on the symbolic and non-symbolic physical size comparison task, c error rates on the symbolic and non-symbolic numerical comparison task, $\mathbf{d}$ error rates on the symbolic and nonsymbolic physical size comparison task. The results are divided by congruency (congruent, neutral and incongruent). Note that neutral is not present in the nonsymbolic number comparison task because it is not possible to create a neutral condition while taking into account the effects of the visual stimulus properties
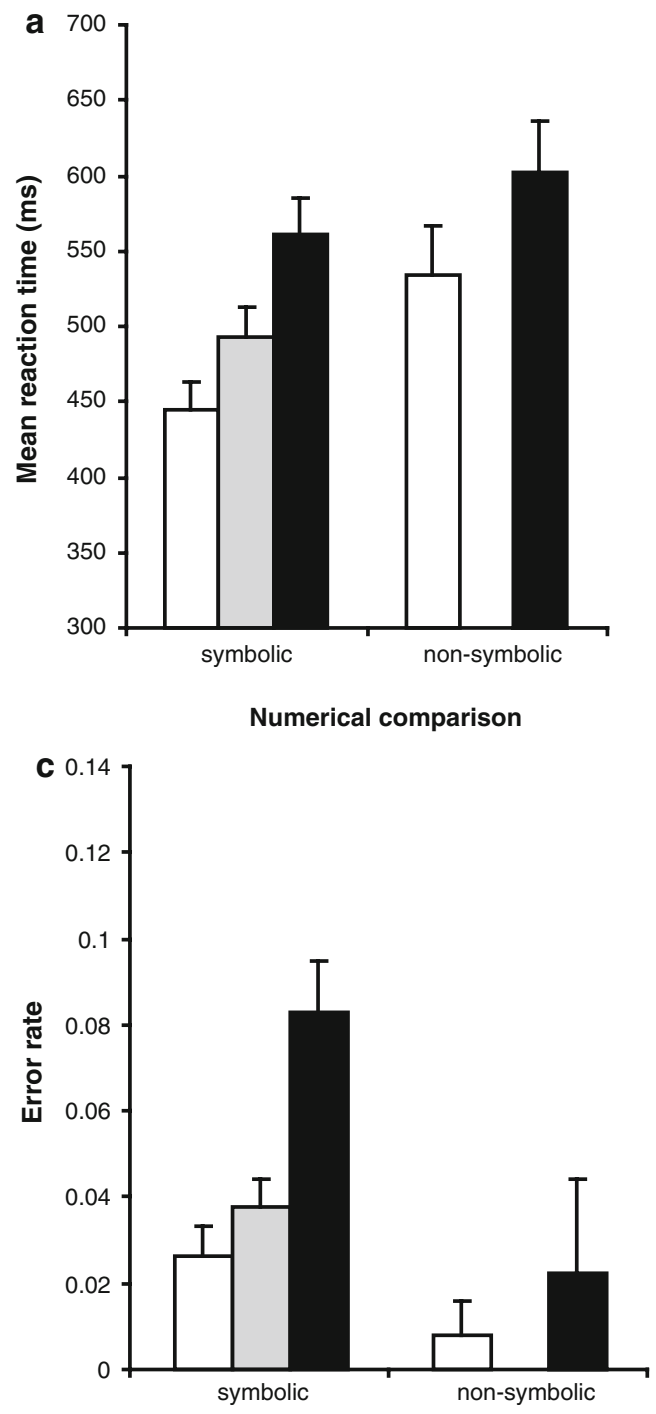

Physical size comparison
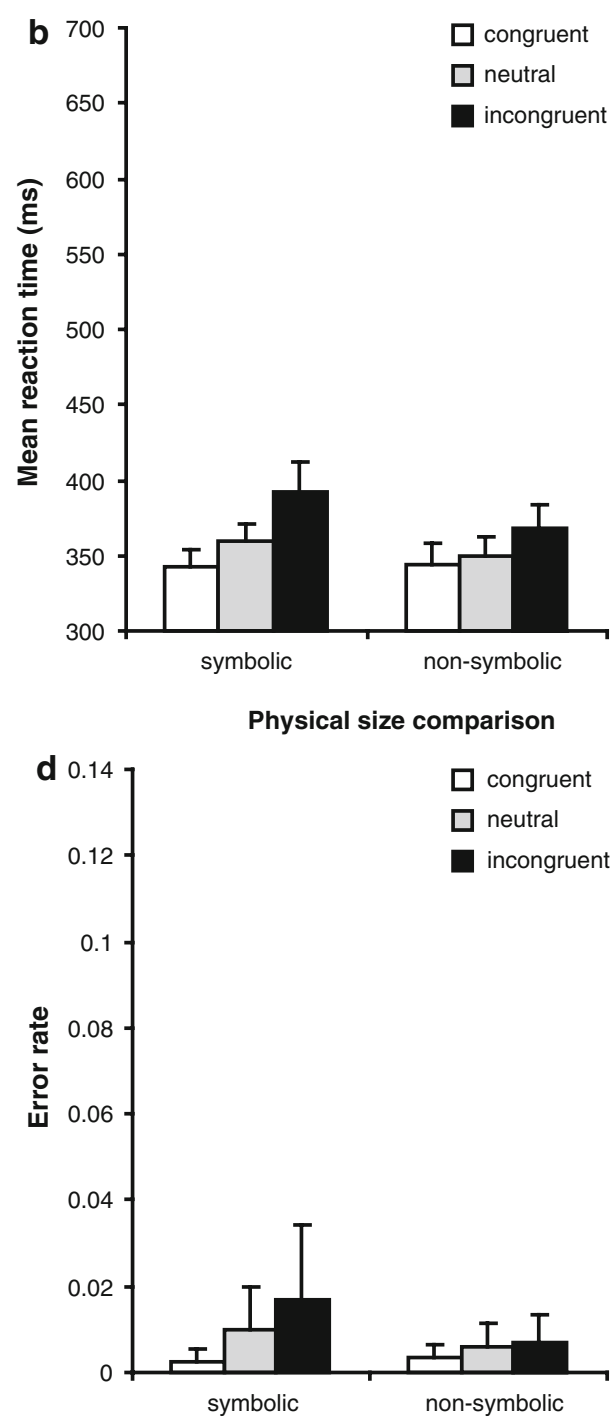

well as a significant interference effect of $64 \mathrm{~ms}[F(1$, 14) $=80.29, P<0.001]$ was present. In addition, the participants made only significantly more errors in the incongruent $(4 \%)[F(1,14)=8.66, P<0.02]$ compared to the neutral condition.

\section{Size comparison}

The simple main effect for notation did not reveal a significant difference for reaction times $[F<1]$ or error rates $[F<1]$ indicating that both size comparison tasks were of similar difficulty. In addition, the simple main effect for congruency revealed a significant effect for both reaction time $[F(1,14)=23.62, P<0.001]$ and error rate $[F(1$, $14)=19.82, P<0.001]$. The adults responded slower and made more errors in the incongruent $(376 \mathrm{~ms}, 4 \%)$ compared to the congruent condition ( $341 \mathrm{~ms}, 1 \%$ errors).
The SCE was further analyzed with a one-way ANOVA in order to examine the facilitation and interference components. The results showed that only the main effect for congruency was significant for reaction time $[F(2,26)=$ 20.90, $P<0.001]$ and error rate $[F(2,26)=17.34$, $P<0.001$ ] (see Fig. 2b, d). When both congruency conditions were compared to the neutral condition it appeared that the facilitation $(11 \mathrm{~ms})$ effect $[F(1,14)=22.87$, $P<0.001]$ and the interference $(25 \mathrm{~ms})$ effect $[F(1,14)=$ 16.70, $P=0.001]$ were significant. In addition, the participants made significantly fewer errors $(1 \%)$ in the congruent $[F(1,14)=4.78, P<0.05]$ and more errors $(3 \%)$ in the incongruent condition $[F(1,14)=17.57$, $P=0.001]$ when compared to the neutral condition.

Overall, it can be concluded that the symbolic and nonsymbolic comparison task were of equal difficulty in the size comparison condition. In addition, the results of the 
adults of both the numerical and the physical size congruity tasks revealed a SCE. More importantly, under each task the SCE pattern was similarly independent of the notation. Thus the non-symbolic tasks measure automatic quantity processing in the same manner as their symbolic counter parts.

\section{Results of the child study}

The two-way ANOVA revealed a significant congruency effect $[F(2,28)=16.18, P<0.001]$ as well as an interaction effect between notation and congruency $[F(2$, $28)=19.23, P<0.001]$ (see Fig. 3a). To further our understanding regarding the source of the two-way interaction, we conducted simple effects analyses for symbolic and non-symbolic notation separately (Keppel 1991).

Symbolic notation

The simple main effect for congruency did not reveal a significant effect for reaction time $[F(2,28)=0.018$, $P=0.98]$ (see Fig. 3a) or error rate $[F(2,28)=2.68$, $P=0.12$ ] (see Fig. 3b).

\section{Non-symbolic notation}

The simple main effect for congruency was significant for reaction time $[F(2,28)=26.4, P<0.001]$ (see Fig. 3a) but not for error rate $[F(2,28)=2.79, P=0.12]$ (see Fig. 3b). When both congruency conditions were compared to the neutral condition it appeared that the facilitation (40 ms) effect $[F(1,14)=5.27, P<0.05]$ and the interference $(93 \mathrm{~ms})$ effect $[F(1,14)=31.07, P<0.001]$ were significant. To exclude the possibility of large inter-individual differences, we also looked at the individual response patterns and found that only one subject did not reveal an interference effect and three subjects did not reveal a facilitation effect.

The interference effect compared between adults and children

In addition, we compared the performance of both children and adults on the symbolic and non-symbolic size comparison task on the basis of the SCE. In order to deal with the overall slower responses of the children, we standardized the data by subtracting the median reaction times of the congruent from the incongruent trials (i.e., the SCE), and divided the difference by the neutral condition for each participant (Cohen Kadosh et.al 2007a). This standardized SCE factor was entered into a two-way ANOVA with notation (symbolic, non-symbolic) and group (adults, children). The two-way interaction of notation and group was significant $[F(1,56)=23.13, P<0.001]$ (see Fig. 4).

\section{Symbolic notation}

The simple effects analysis for symbolic notation showed a larger standardized SCE effect for the adults (10) than the children $(1)[F(1,56)=10.57, P=0.001]$.

Non-symbolic notation

The simple effects analysis for non-symbolic notation showed a larger standardized interference effect for the children (15) than for the adults $(5)[F(1,56)=12.60$, $P<0.001]$.
Fig. 3 The performance of the children on the symbolic and non-symbolic size congruity tasks. a Mean reaction time on the symbolic and non-symbolic numerical comparison task, b error rates on the symbolic and non-symbolic numerical comparison task. The results are divided by congruency (congruent, neutral and incongruent). Note that the scale is different than the one used for the adult studies
Physical size comparison



Physical size comparison

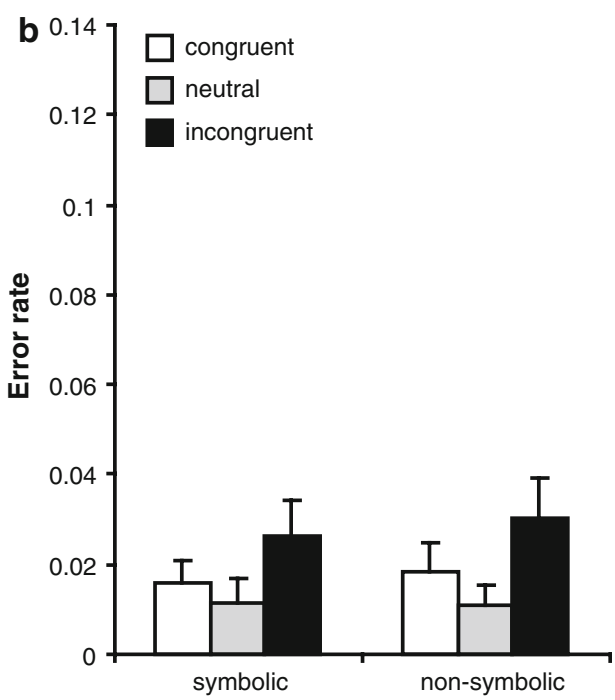




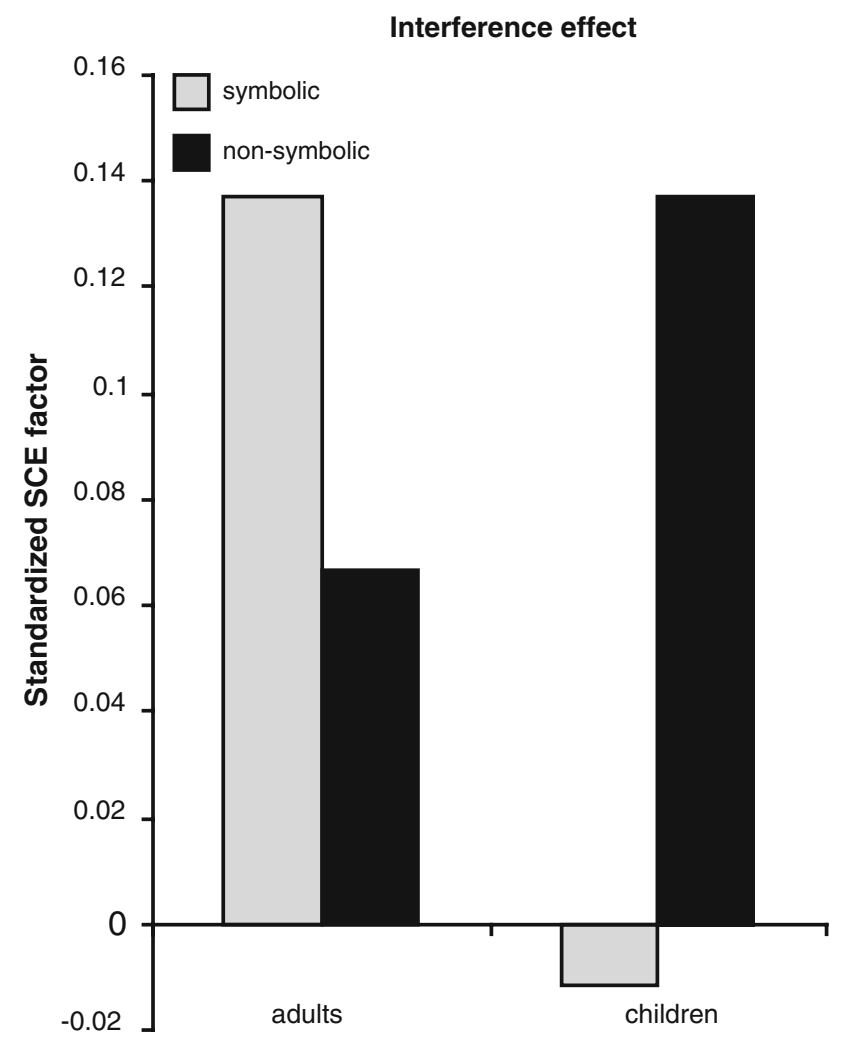

Fig. 4 The standardized interference component of the mean reaction times of the adults and the children on the symbolic and non-symbolic physical size congruity task

In conclusion, the adults revealed a larger SCE than the children in the symbolic notation task, which is in accordance with the previous finding that no significant SCE was present for children in this task. In the non-symbolic notation task a larger SCE was obtained for children than for adults.

\section{Discussion}

Previous studies have revealed that automatic access to numbers is present in third graders but not in children (at the beginning) of the first grade. In this study, we intended to get further insight in the ability of 5-year-old children to map numbers automatically on the mental number line. First, we asked adults to perform a symbolic and nonsymbolic size congruity task by making a numerical and physical size judgment. Second, the children judged physical size in the symbolic and non-symbolic size congruity tasks. In the adult study, the presence of a SCE for non-symbolic stimuli in the physical and numerical judgment task would indicate that the non-symbolic task is a suitable measure to study automatic processing of number and physical size. The results of the child study would subsequently give further insight in the development of symbolic and non-symbolic number processing.

For the adults, a SCE effect was present for both the symbolic and non-symbolic numerical judgment task. This finding indicates that the participants were able to decide which array contained a larger number of dots, even in the incongruent condition where the visual properties (i.e., continuous variables) were misleading. This result also suggests that the participants had automatic access to physical size which is consistent with the finding of Hurewitz et al. (2006). The SCE effect was also present in the relatively easier physical size judgment tasks. Faster responses were obtained when numerosity was congruent with physical size and slower responses were obtained when numerosity was incongruent with physical size. The presence of a SCE in the non-symbolic size comparison task indicates that the participants processed numerosity automatically even though they were instructed to attend the more prominent physical size. Thus, number and physical size interfered with each other in a similar manner irrespective of the notation they were presented in. Therefore, the non-symbolic physical size and numerical judgment tasks are a suitable way to investigate numerosity processing in children.

For the children, no SCE was present in the symbolic size congruity task which is in accordance with previous studies (Berch et al. 1999; Girelli et al. 2000; Rubinsten et al. 2002). At the age of five, children are familiar with the number symbols but do not have automatic access to their meaning. However, in the non-symbolic task the children revealed a SCE, indicating that the children had direct access to non-symbolic numerosities although it was not part of the task requirement. These results also show that the children had knowledge about both, physical size and numerical value. Faster responses in the congruent and slower responses in the incongruent condition, when compared to the neutral condition, could only arise if the children have knowledge about the two magnitudes and the fact that they can be in agreement or in conflict with each other. Therefore, the absence of a SCE in the symbolic task appears to be unrelated to a lack of mathematical or cognitive abilities necessary for the task, but instead related to the relative infamiliarity with the number symbols. In line with this result, Butterworth et al. (2001) suggested that repeated exposure to the number symbols will lead to automatic processing of numbers which in turn is considered necessary to become skilled in mathematics.

Children also revealed a larger interference effect than adults on the non-symbolic size comparison task. This finding is in agreement with the contemporary findings that children have immature inhibitory mechanisms, meaning that they are less capable of suppressing the irrelevant features of the stimulus (in the current case, numerosity) 
(Leon-Carrion et al. 2004; Schroeter et al. 2004). The fact that children are less capable of suppressing the irrelevant feature should be taken into account in the paradigms used in the infants studies. Younger children will be more prone to attend to the prominent continuous variables instead of number in comparison to adults and are therefore less likely to be aware of a possible change in numerosity. The more prominent the continuous variables are, the less likely it is that the child is going to perceive a change in numerosity especially when they are not instructed to attend to numerosity changes.

In contrast to Hurewitz et al. (2006), we found a SCE in the non-symbolic physical size judgment condition for both adults and children. Hurewitz et al. (2006) explained the absence of a congruency effect with the relative speed of processing account (Schwarz and Ischebeck 2003), which states that area is processed faster and therefore not susceptible to the influence of the slower numerical processes. Hurewitz et al. (2006) concluded that the faster processing of physical size compared to numerosity might be the origin of the conflicting results in infant studies. But from the results they presented (cf. Figs. 3, 4) it can be concluded that the participants were faster to judge numerosity (mean reaction times run from approximately 450-700) compared to area (mean reaction times run from approximately 700-800) which contradicts their reasoning. An alternative explanation for the lack of a SCE effect in the Hurewitz et al. (2006) study could be related to the fact that they used numerosities both above and below the subitizing range (3-9). For the small distance conditions, this results in number pairs that are difficult to discriminate (e.g., 6-7, 8-9 and 7-8; note this is $30 \%$ of the trials). Interestingly, the large error rate that was present in the small distance, incongruent condition, of their number judgment task was $30 \%$ as well. Therefore, it appears plausible that in their study the participants were unable to judge numerosity on specific trials which therefore did not lead to interference when physical size has to be judged.

The symbolic and non-symbolic Stroop task presented in this study is also a good paradigm to study the debate about the mechanisms underlying dyscalculia. Automatic access to symbolic numbers has been proposed to be affected in dyscalculics. Dyscalculic participants performing a physical size comparison of Arabic numbers revealed no facilitation effects (Rubinsten and Henik 2005). Moreover, this lack of a facilitation effect is also obtained in normal subjects when the right intraparietal sulcus (IPS), the area expected to subserve number processing, was stimulated using transcranial magnetic stimulation (TMS) (Cohen Kadosh et al. 2007b). Interestingly, when the relative speed of processing of numbers and physical size are accounted for in the task, facilitation effects were obtained (Rousselle and Noel 2007). See for an alternative explanation Mussolin and Noel (2007). These results together suggest a prominent role for automatic mapping of numbers on the mental number line in number processing. The idea of impaired automatization processes is in line with Wilson and colleagues who proposed two possible deficits that could be the cause of dyscalculia: (1) a deficit in number sense or non-symbolic representation of number or (2) a disconnection between symbolic and non-symbolic representations (Rubinsten and Henik 2005; Wilson et al. 2006a, b; Wilson et al. 2006b). Cohen Kadosh and colleagues suggested that dyscalculia is not only limited to a problem in the numerical domain but instead is a deficit in general magnitude representation, meaning that also nonnumerical processes can be affected (Cohen Kadosh et al. 2007b; Cohen Kadosh and Walsh 2007). In contrast, problems in a cognitive domain have also been proposed as the source of dyscalculia (Geary 1993). The two Stroop tasks presented in this study allow direct comparison between automatic symbolic and non-symbolic processes of numbers on one hand, and non-numerical magnitude processing on the other hand, while excluding the possibility that different outcomes on both tasks are related to distinct task requirements (e.g., mathematical or cognitive processes). In this manner, these tasks might become a valuable tool in dyscalculia research, even before children acquire a more formal education of numbers.

To conclude, we presented a non-symbolic size congruity task that gave more insight into symbolic and nonsymbolic quantity processing in adults and children. Already at the age of five, children who perform well on mathematics automatically process numerosities even while they are instructed to attend to physical size. Their inability to process Arabic numerals automatically is unrelated to the mathematical abilities required for the task but instead seems to be related to an immature link between the number symbol and the magnitude it represents.

Acknowledgments We would like to thank Maarten J. van der Smagt for helpful comments on a previous version of this manuscript. This research was supported by NWO Grant 051.04 .050 of the Cognition Program of the Netherlands Organization for Scientific Research (NWO). RCK is supported by Marie Curie Intra European Fellowship.

Open Access This article is distributed under the terms of the Creative Commons Attribution Noncommercial License which permits any noncommercial use, distribution, and reproduction in any medium, provided the original author(s) and source are credited.

\section{References}

Algom D, Dekel A, Pansky A (1996) The perception of number from the separability of the stimulus: the Stroop effect revisited. Mem Cognit 24(5):557-572 
Barth H, Kanwisher N, Spelke E (2003) The construction of large number representations in adults. Cognition 86(3):201-221. doi: 10.1016/S0010-0277(02)00178-6

Barth H, La Mont K, Lipton J, Spelke ES (2005) Abstract number and arithmetic in preschool children. Proc Natl Acad Sci USA 102(39):14116-14121. doi:10.1073/pnas.0505512102

Barth H, La Mont K, Lipton J, Dehaene S, Kanwisher N, Spelke E (2006) Non-symbolic arithmetic in adults and young children. Cognition 98(3):199-222. doi:10.1016/j.cognition.2004.09.011

Berch DB, Foley EJ, Hill RJ, Ryan PM (1999) Extracting parity and magnitude from Arabic numerals: developmental changes in number processing and mental representation. J Exp Child Psychol 74(4):286-308. doi:10.1006/jecp. 1999.2518

Brannon EM (2002) The development of ordinal numerical knowledge in infancy. Cognition 83(3):223-240. doi:10.1016/S00100277(02)00005-7

Brannon EM, Abbott S, Lutz DJ (2004) Number bias for the discrimination of large visual sets in infancy. Cognition 93(2):B59-B68. doi:10.1016/j.cognition.2004.01.004

Butterworth B, Zorzi M, Girelli L, Jonckheere AR (2001) Storage and retrieval of addition facts: the role of number comparison. Q J Exp Psychol A 54(4):1005-1029. doi:10.1080/ 02724980143000064

Cohen Kadosh R, Walsh V (2007) Dyscalculia. Curr Biol 17(22):R946-R947. doi:10.1016/j.cub.2007.08.038

Cohen Kadosh R, Cohen Kadosh K, Linden DE, Gevers W, Berger A, Henik A (2007a) The brain locus of interaction between number and size: a combined functional magnetic resonance imaging and event-related potential study. J Cogn Neurosci 19(6):957-970. doi:10.1162/jocn.2007.19.6.957

Cohen Kadosh R, Cohen Kadosh K, Schuhmann T, Kaas A, Goebel R, Henik A et al (2007b) Virtual dyscalculia induced by parietallobe TMS impairs automatic magnitude processing. Curr Biol 17(8):689-693. doi:10.1016/j.cub.2007.02.056

Dehaene S (1992) Varieties of numerical abilities. Cognition 44(12):1-42. doi:10.1016/0010-0277(92)90049-N

Dehaene S (2003) The neural basis of the Weber-Fechner law: a logarithmic mental number line. Trends Cogn Sci 7(4):145-147. doi:10.1016/S1364-6613(03)00055-X

Dehaene S, Bossini S, Giraux P (1993) The mental representation of parity and numerical magnitude. J Exp Psychol Gen 122:371-396

Feigenson L (2005) A double-dissociation in infants representations of object arrays. Cognition 95(3):B37-B48. doi:10.1016/ j.cognition.2004.07.006

Fischer MH, Warlop N, Hill RL, Fias W (2004) Oculomotor bias induced by number perception. Exp Psychol 51(2):91-97. doi: 10.1027/1618-3169.51.2.91

Gallistel CR, Gelman R (1992) Preverbal and verbal counting and computation. Cognition 44(1-2):43-74. doi:10.1016/0010-0277 (92) $90050-\mathrm{R}$

Geary DC (1993) Mathematical disabilities: cognitive, neuropsychological, and genetic components. Psychol Bull 114(2):345-362. doi:10.1037/0033-2909.114.2.345

Gevers W, Lammertyn J, Notebaert W, Verguts T, Fias W (2006) Automatic response activation of implicit spatial information: evidence from the SNARC effect. Acta Psychol (Amst) 122(3):221-233. doi:10.1016/j.actpsy.2005.11.004

Girelli L, Lucangeli D, Butterworth B (2000) The development of automaticity in accessing number magnitude. J Exp Child Psychol 76(2):104-122. doi:10.1006/jecp. 2000.2564

Henik A, Tzelgov J (1982) Is three greater than five: the relation between physical and semantic size in comparison tasks. Mem Cognit 10(4):389-395

Huntley-Fenner G (2001) Children's understanding of number is similar to adults and rats: numerical estimation by $5-7$-year-olds. Cognition 78(3):B27-B40. doi:10.1016/S0010-0277(00)00122-0
Hurewitz F, Gelman R, Schnitzer B (2006) Sometimes area counts more than number. Proc Natl Acad Sci USA 103(51):1959919604. doi:10.1073/pnas.0609485103

Jordan KE, Brannon EM (2006) The multisensory representation of number in infancy. Proc Natl Acad Sci USA 103(9):3486-3489. doi:10.1073/pnas.0508107103

Kaufmann L, Koppelstaetter F, Siedentopf C, Haala I, Haberlandt E, Zimmerhackl LB et al (2006) Neural correlates of the numbersize interference task in children. Neuroreport 17(6):587-591. doi:10.1097/00001756-200604240-00007

Keppel G (1991) Design and analysis. A researcher's handbook. Prentice Hall, Upper Saddle River

Leon-Carrion J, Garcia-Orza J, Perez-Santamaria FJ (2004) Development of the inhibitory component of the executive functions in children and adolescents. Int J Neurosci 114(10):1291-1311. doi:10.1080/00207450490476066

Lipton JS, Spelke ES (2005) Preschool children's mapping of number words to nonsymbolic numerosities. Child Dev 76(5):978-988. doi:10.1111/j.1467-8624.2005.00891.x

Mix KS, Huttenlocher J, Levine SC (2002) Multiple cues for quantification in infancy: is number one of them? Psychol Bull 128(2):278-294. doi:10.1037/0033-2909.128.2.278

Mussolin C, Noel MP (2007) The nonintentional processing of Arabic numbers in children. J Clin Exp Neuropsychol 29(3):225-234. doi:10.1080/13803390600629759

Rousselle L, Noel MP (2007) Basic numerical skills in children with mathematics learning disabilities: a comparison of symbolic vs non-symbolic number magnitude processing. Cognition 102(3):361-395. doi:10.1016/j.cognition.2006.01.005

Rousselle L, Palmers E, Noel MP (2004) Magnitude comparison in preschoolers: what counts? Influence of perceptual variables. J Exp Child Psychol 87(1):57-84. doi:10.1016/j.jecp. 2003.10.005

Rubinsten O, Henik A (2005) Automatic activation of internal magnitudes: a study of developmental dyscalculia. Neuropsychology 19(5):641-648. doi:10.1037/0894-4105.19.5.641

Rubinsten O, Henik A, Berger A, Shahar-Shalev S (2002) The development of internal representations of magnitude and their association with Arabic numerals. J Exp Child Psychol 81(1):74-92. doi:10.1006/jecp. 2001.2645

Schroeter ML, Zysset S, Wahl M, von Cramon DY (2004) Prefrontal activation due to Stroop interference increases during development-an event-related fNIRS study. Neuroimage 23(4):13171325. doi:10.1016/j.neuroimage.2004.08.001

Schwarz W, Heinze HJ (1998) On the interaction of numerical and size information in digit comparison: a behavioral and eventrelated potential study. Neuropsychologia 36(11):1167-1179. doi:10.1016/S0028-3932(98)00001-3

Schwarz W, Ischebeck A (2003) On the relative speed account of number-size interference in comparative judgments of numerals. J Exp Psychol Hum Percept Perform 29(3):507-522. doi: 10.1037/0096-1523.29.3.507

Szucs D, Soltesz F (2007) Event-related potentials dissociate facilitation and interference effects in the numerical Stroop paradigm. Neuropsychologia 45(14):3190-3202. doi:10.1016/ j.neuropsychologia.2007.06.013

Szucs D, Soltesz F, Jarmi E, Csepe V (2007) The speed of magnitude processing and executive functions in controlled and automatic number comparison in children: an electro-encephalography study. Behav Brain Funct 30:3-23. doi:10.1186/1744-9081-323

Temple E, Posner MI (1998) Brain mechanisms of quantity are similar in 5-year-old children and adults. Proc Natl Acad Sci USA 95(13):7836-7841. doi:10.1073/pnas.95.13.7836

Tzelgov J, Henik A, Berger J (1992) Controlling Stroop effects by manipulating expectations for color words. Mem Cognit 20(6):727-735 
Verguts T, Fias W, Stevens M (2005) A model of exact small-number representation. Psychon Bull Rev 12(1):66-80

Wilson AJ, Dehaene S, Pinel P, Revkin SK, Cohen L, Cohen D (2006) Principles underlying the design of "The Number Race", an adaptive computer game for remediation of dyscalculia. Behav Brain Funct 30:2-19. doi:10.1186/1744-9081-2-19

Wilson AJ, Revkin SK, Cohen D, Cohen L, Dehaene S (2006) An open trial assessment of "The Number Race", an adaptive computer game for remediation of dyscalculia. Behav Brain Funct 30:2-20. doi:10.1186/1744-9081-2-20

Xu F, Spelke ES (2000) Large number discrimination in 6-month-old infants. Cognition 74(1):B1-B11. doi:10.1016/S0010-0277(99) 00066-9
Xu F, Spelke ES, Goddard S (2005) Number sense in human infants. Dev Sci 8(1):88-101. doi:10.1111/j.1467-7687.2005.00395.x

Zhou X, Chen Y, Chen C, Jiang T, Zhang H, Dong Q (2007) Chinese kindergartners' automatic processing of numerical magnitude in stroop-like tasks. Mem Cognit 35(3):464-470

Zorzi M, Butterworth B (1999) A computational model of number comparison. In: Hahn M, Stoness SC (eds) Proceedings of the twenty first annual conference of the cognitive science society. Erlbaum, Mahwah, pp 778-783 